\title{
Measured Polarized Spectral Responsivity of JPSS J1 VIIRS Using the NIST T-SIRCUS
}

\author{
Jeff McIntire $^{a}$, James B. Young ${ }^{b}$, David Moyer ${ }^{c}$, Eugene Waluschka ${ }^{d}$, and Xiaoxiong Xiong ${ }^{d}$ \\ ${ }^{a}$ Sigma Space Corporation, Lanham, MD 20706 USA \\ ${ }^{b}$ Stellar Solutions, Palo Alto, CA 94306 USA \\ ${ }^{c}$ The Aerospace Corporation, El Segundo, CA 90245-4691 USA \\ ${ }^{d}$ NASA Goddard Space Flight Center, Greenbelt, MD, 20771 USA
}

\begin{abstract}
Recent pre-launch measurements performed on the Joint Polar Satellite System (JPSS) J1 Visible Infrared Imaging Radiometer Suite (VIIRS) using the NIST T-SIRCUS monochromatic source have provided wavelength dependent polarization sensitivity for select spectral bands and viewing conditions. Measurements were made at a number of input linear polarization states (twelve in total) and initially at thirteen wavelengths across the bandpass (later expanded to seventeen for some cases). Using the source radiance information collected by an external monitor, a spectral responsivity function was constructed for each input linear polarization state. Additionally, an unpolarized spectral responsivity function was derived from these polarized measurements. An investigation of how the centroid, bandwidth, and detector responsivity vary with polarization state was weighted by two model input spectra to simulate both ground measurements as well as expected on-orbit conditions. These measurements will enhance our understanding of VIIRS polarization sensitivity, improve the design for future flight models, and provide valuable data to enhance product quality in the post-launch phase.
\end{abstract}

Keywords: JPSS, S-NPP, VIIRS, polarization, calibration

\section{INTRODUCTION}

Pre-launch instrument level characterization of the Joint Polar Satellite System (JPSS) J1 Visible Infrared Imaging Radiometer Suite (VIIRS) instrument was completed in January 2015, with launch scheduled for late 2016. ${ }^{1}$ Ambient testing performed in late 2013 revealed that some spectral bands were more sensitive to polarized light than expected (in particular the bands centered at 412, 445, 488, and $555 \mathrm{~nm}$, hereafter referred to as M1, M2, M3, and M4). ${ }^{2}$ Additional testing was conducted in late 2014, including limited monochromatic measurements of M1 and M4. ${ }^{3,4}$ These monochromatic measurements made use of tunable lasers provided and operated by NIST and NASA known as T-SIRCUS (Traveling Spectral Irradiance and Radiance Responsivity Calibrations Using Uniform Sources). ${ }^{5}$ The T-SIRCUS laser system and general test setup used were discussed in previous work, ${ }^{3,4}$ and will not be repeated here. A list of all the monochromatic measurements made is provided in Table 1 for all test configurations including half angle mirror (HAM) side and telescope scan angle. These measurements were used to validate the spectral behavior of the polarization sensitivity as described by ray trace modeling developed by Raytheon ${ }^{3}$ as well as to compare to broadband measurements. ${ }^{2}$

In addition, the T-SIRCUS polarization measurements were used to create spectral response functions of the VIIRS instrument under limited conditions. The sensor response was recorded for each of the wavelengths listed in Table 1 for twelve different linear polarization states and the corresponding T-SIRCUS radiance was recorded in real-time. From this data, a separate spectral response function was constructed for each polarization state. The variation of some spectral band characteristics with polarization state (such as centroid, bandwidth, and responsivity) was investigated. Each of the spectral response functions were also convolved with different input spectra, to investigate how sensitive the spectral band characteristics were to both pre-launch and on-orbit conditions.

Send correspondence to Jeff McIntire: jeffrey.mcintire@ssaihq.com 
Table 1. T-SIRCUS polarization measurements performed as a function of wavelength, HAM side, scan angle, and test type (stray light, polarizer efficiency, or polarization sensitivity). Wavelengths were rounded to the nearest nm.

\begin{tabular}{|c|c|c|c|c|}
\hline Test Type & HAM Side & $\begin{array}{l}\text { Scan Angle } \\
\text { [degrees] }\end{array}$ & Target Band & $\begin{array}{l}\text { Wavelengths } \\
{[\mathrm{nm}]}\end{array}$ \\
\hline Stray Light - Dark & 1 & -8 & M1, M4 & $\mathrm{NA}$ \\
\hline Stray Light - Lollipop & 1 & -8 & $\begin{array}{l}\text { M1 } \\
\text { M4 }\end{array}$ & $\begin{array}{l}415 \\
559\end{array}$ \\
\hline $\begin{array}{l}\text { Polarizer } \\
\text { Efficiency }\end{array}$ & 1 & -8 & $\begin{array}{l}\text { M1 } \\
\text { M4 }\end{array}$ & $\begin{array}{l}401,412,420 \\
559\end{array}$ \\
\hline $\begin{array}{l}\text { Polarization } \\
\text { Sensitivity }\end{array}$ & 1 & -8 & M1 & $\begin{array}{l}397,400,402,404,406,408,410,413,415, \\
417,419,421,424\end{array}$ \\
\hline $\begin{array}{l}\text { Polarization } \\
\text { Sensitivity }\end{array}$ & 1 & +45 & M1 & $\begin{array}{l}397,399,402,404,406,408,410,413,415 \\
417,419,421,424\end{array}$ \\
\hline $\begin{array}{l}\text { Polarization } \\
\text { Sensitivity }\end{array}$ & 1 & -8 & M4 & $\begin{array}{l}543,546,547,548,550,552,553,555,556, \\
558,560,561,562,564,567,569,572\end{array}$ \\
\hline $\begin{array}{l}\text { Polarization } \\
\text { Sensitivity }\end{array}$ & 0 & -8 & M4 & $\begin{array}{l}543,545,547,548,550,553,556,559,562 \\
564,567,569,572\end{array}$ \\
\hline $\begin{array}{l}\text { Polarization } \\
\text { Sensitivity }\end{array}$ & 1 & +45 & M4 & $\begin{array}{l}543,545,547,549,551,552,553,554,556, \\
558,559,561,562,564,567,569,572\end{array}$ \\
\hline
\end{tabular}

\section{ABSOLUTE SPECTRAL RESPONSE}

The monochromatic polarization sensitivity data was used to construct absolute spectral response $(A S R)$ functions. ${ }^{6}$ The $A S R$ are wavelength dependent functions of the the ratio of the offset corrected sensor response to the radiance entering the VIIRS telescope aperture, or

$$
A S R(\lambda, \alpha)=\frac{d n(\lambda, \alpha)}{L(\lambda, \alpha)}
$$

where $\lambda$ is the wavelength and $\alpha$ is the polarization state at the telescope aperture. Note that the units on $A S R$ are $\left[d n /\left(W / m^{2} / s r\right)\right] . \alpha=0$ is the polarization state of light entering the VIIRS aperture for which the $E$ field is perpendicular to the VIIRS scan plane, and that the polarization angle rotates from 0 to 180 degrees clockwise as seen from VIIRS (data was collected at every 15 degrees).

The radiance monitor was mounted directly to the NIST integrating sphere, and does not incorporate the transmittance of the polarizer sheet. ${ }^{3}$ In order to determine the true radiance at VIIRS aperture, the unpolarized $A S R$ determined from polarization testing is ratioed to the $A S R$ developed from spectral testing with the T-SIRCUS. ${ }^{7} A S R$ functions were constructed for each of the twelve input polarization states (as well as an unpolarized case) for each of the five polarization sensitivity cases listed in Table 1.

For some wavelength / polarizer angle combinations, no valid data was collected. ${ }^{4}$ In these cases, the Fourier analysis $^{4}$ was used to predict the sensor response at the wavelength / polarizer angle of the missing data. In addition, the zeroth order Fourier component is effectively the unpolarized sensor response, and was used to construct the unpolarized $A S R$.

By integrating the wavelength dependent $A S R$ over the extended bandpass, the detector responsivity is 
(a)

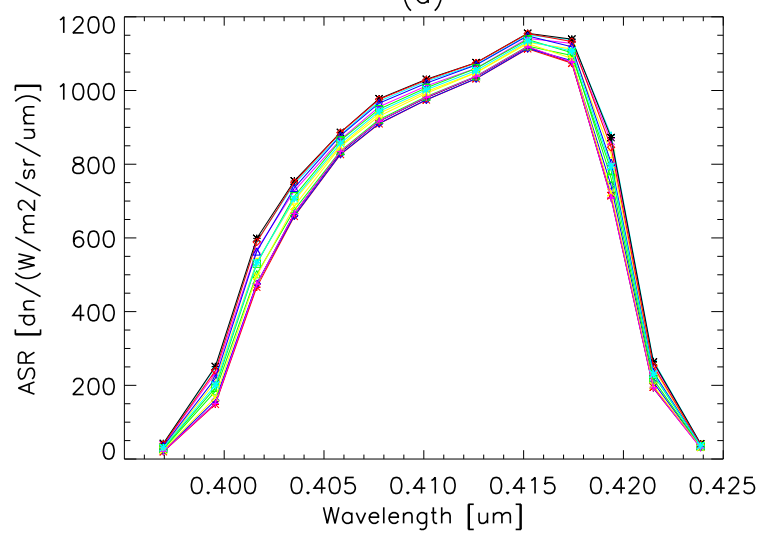

(b)

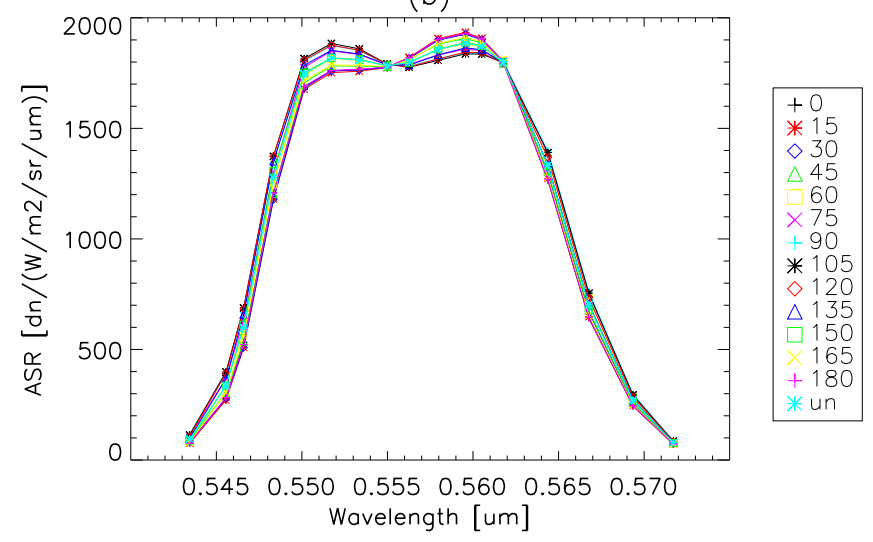

Figure 1. M1 (a) and M4 (b) ASR shown for detector 9. The data used here was collected at -8 degrees scan angle, HAM side 1. The legend defines the different symbol / color combinations which correspond to each polarization state (and "un" refers to the unpolarized $A S R$ ).

determined from the following: ${ }^{6}$

$$
\text { responsivity }(\alpha)=\sum_{n=0}^{N_{\lambda}-1} \frac{\left[A S R\left(\lambda_{n+1}, \alpha\right)+A S R\left(\lambda_{n}, \alpha\right)\right]}{2}\left(\lambda_{n+1}-\lambda_{n}\right),
$$

where $N_{\lambda}$ is the number of wavelengths measured across the bandpass and a trapezoidal integration is used. Note that the wavelength interval is not constant, as seen from Table 1. The units of the detector responsivity are $\left[d n /\left(W / m^{2} / s r / \mu m\right)\right]$. The detector responsivity was determined for each detector, polarization state (as well as the unpolarized case) and test configuration, the results of which were compared to the responsivity measurements using a broadband source. ${ }^{2}$

The $A S R$ was also integrated to determine the band centroid and bandwidth of each polarization angle by ${ }^{6}$

$$
\operatorname{centroid}(\alpha)=\frac{\sum_{n=0}^{N_{\lambda}-1} \frac{\left[\lambda_{n+1} A S R\left(\lambda_{n+1}, \alpha\right)+\lambda_{n} A S R\left(\lambda_{n}, \alpha\right)\right]}{2}\left(\lambda_{n+1}-\lambda_{n}\right)}{\sum_{n=0}^{N_{\lambda}-1} \frac{\left[A S R\left(\lambda_{n+1}, \alpha\right)+A S R\left(\lambda_{n}, \alpha\right)\right]}{2}\left(\lambda_{n+1}-\lambda_{n}\right)},
$$

and

$$
\text { bandwidth }(\alpha)=\frac{1}{\max [\operatorname{ASR}(\lambda, \alpha)]_{\lambda}} \sum_{n=0}^{N_{\lambda}-1} \frac{\left[\operatorname{ASR}\left(\lambda_{n+1}, \alpha\right)+A S R\left(\lambda_{n}, \alpha\right)\right]}{2}\left(\lambda_{n+1}-\lambda_{n}\right) .
$$

Again, trapezoidal integration is used. Note that the bandwidth is equal to the responsivity divided by the maximum $A S R$ over wavelength.

The $A S R$ constructed in Eq. (1) assumes a flat input spectrum. To consider the effects on Eqs. (3) and (4) of different input spectra, the $A S R$ was modified as follows: ${ }^{6}$

$$
A S R^{\prime}(\lambda, \alpha)=A S R(\lambda, \alpha) \frac{L_{\text {source }}(\lambda)}{L_{\text {source }}^{A V G}},
$$

where $L_{\text {source }}$ is an input source spectral radiance and $L_{\text {source }}^{A V G}$ is the average source spectral radiance, or

$$
L_{\text {source }}^{A V G}=\frac{\sum_{n=0}^{N_{\lambda}-1} \frac{\left[L_{\text {source }}\left(\lambda_{n+1}\right) A S R\left(\lambda_{n+1}, \alpha\right)+L_{\text {source }}\left(\lambda_{n}\right) A S R\left(\lambda_{n}, \alpha\right)\right]}{2}\left(\lambda_{n+1}-\lambda_{n}\right)}{\sum_{n=0}^{N_{\lambda}-1} \frac{\left[A S R\left(\lambda_{n+1}, \alpha\right)+A S R\left(\lambda_{n}, \alpha\right)\right]}{2}\left(\lambda_{n+1}-\lambda_{n}\right)} .
$$


(a)

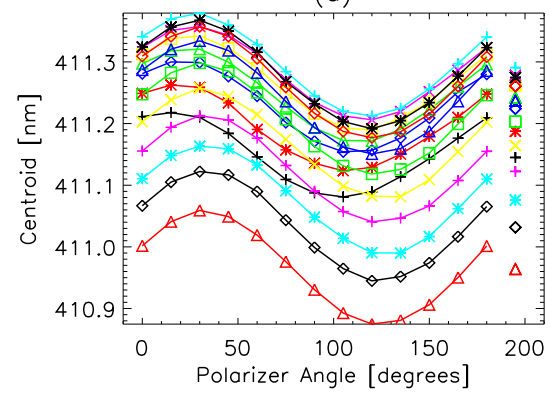

(c)

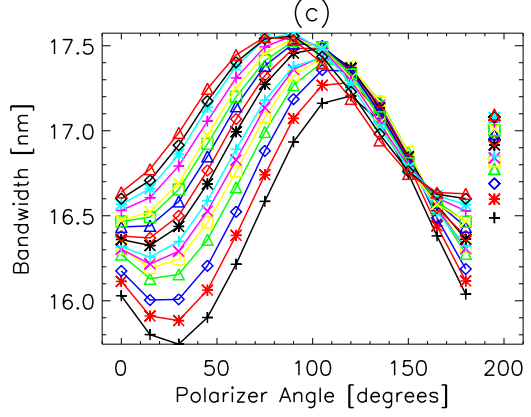

(b)

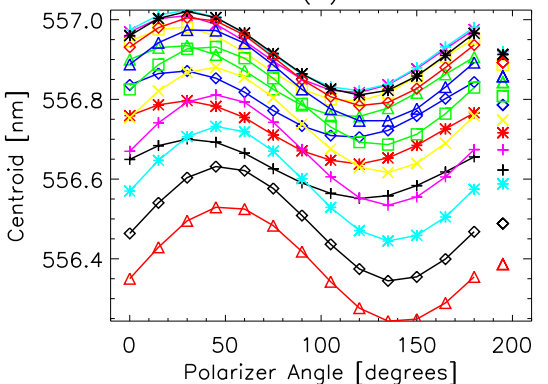

(d)

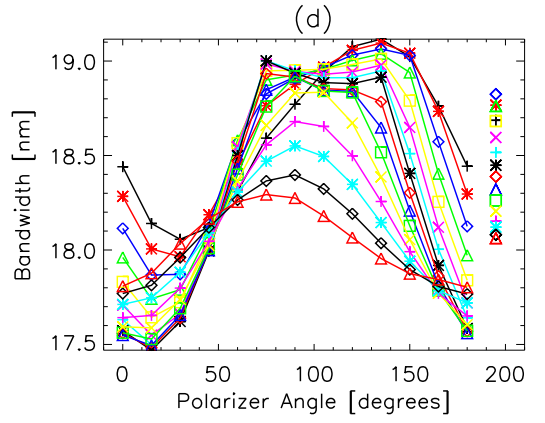

Figure 2. Plots are shown of the band centroid for M1 (a), the band centroid for M4 (b), the bandwidth for M1 (c), and the bandwidth for M4 (d) versus polarization state. All data shown was collected at -8 degrees scan angle, HAM side 1. The legend defines the different symbol / color combinations which correspond to each detector. Note that the unconnected data at 195 degrees in each plot represents the centroid or bandwidth derived from unpolarized data.

By ratioing to the average source spectral radiance, the $A S R^{\prime}$ is only modified by the relative shape of the source spectrum. In this work, two input spectra were considered: the top of the atmosphere (TOA) and spherical integrating source (SIS). The TOA spectrum provides some indication of the expected on-orbit behavior and the SIS spectrum connects these measurements to the pre-launch characterization. Note that in this formulation, the responsivities derived from $A S R$ and $A S R^{\prime}$ are the same the areas under the $A S R$ and $A S R^{\prime}$ curves are equal due to the normalization in Eq. (5) to $L_{\text {source }}^{A V G}$ ].

\section{RESULTS}

The flat spectrum $A S R$ functions derived from the M1 and M4 detector 9, -8 degrees scan angle, HAM side 1 data are shown in Figures 1(a) and (b) for all measured polarization states along with the unpolarized $A S R$ function. Note that although the spectral bands were under-sampled, the general shape of the bandpass is well described. ${ }^{7}$ As a measure of repeatability, 0 and 180 degrees polarization angles were treated separately; the two measurements generally agreed to within $1 \%$, but could disagree by up to $3 \%$ in the lowest response points. There is some variability between the different polarization states on the sides of the M1 and M4 bandpasses and also in the structure of the center of the M4 bandpass. These features are largely responsible for the higher than expected band average polarization sensitivity observed in broadband testing. ${ }^{2}$ The $A S R$ constructed for the remaining detectors and measurements show similar variability, although there is a fair amount of detector dependence.

The variations observed in the center of the bandpass for M4 correspond to a spectral region where a phase shift occurs. ${ }^{4}$ Analysis has shown that a similar phase shift pattern occurs in the same spectral region for Day Night Band (DNB) data, indicating that this phenomena is not caused by the spectral band filters. Ray trace modeling conducted by Raytheon did not predict this behavior; ${ }^{3}$ the root cause of this behavior is under investigation. 

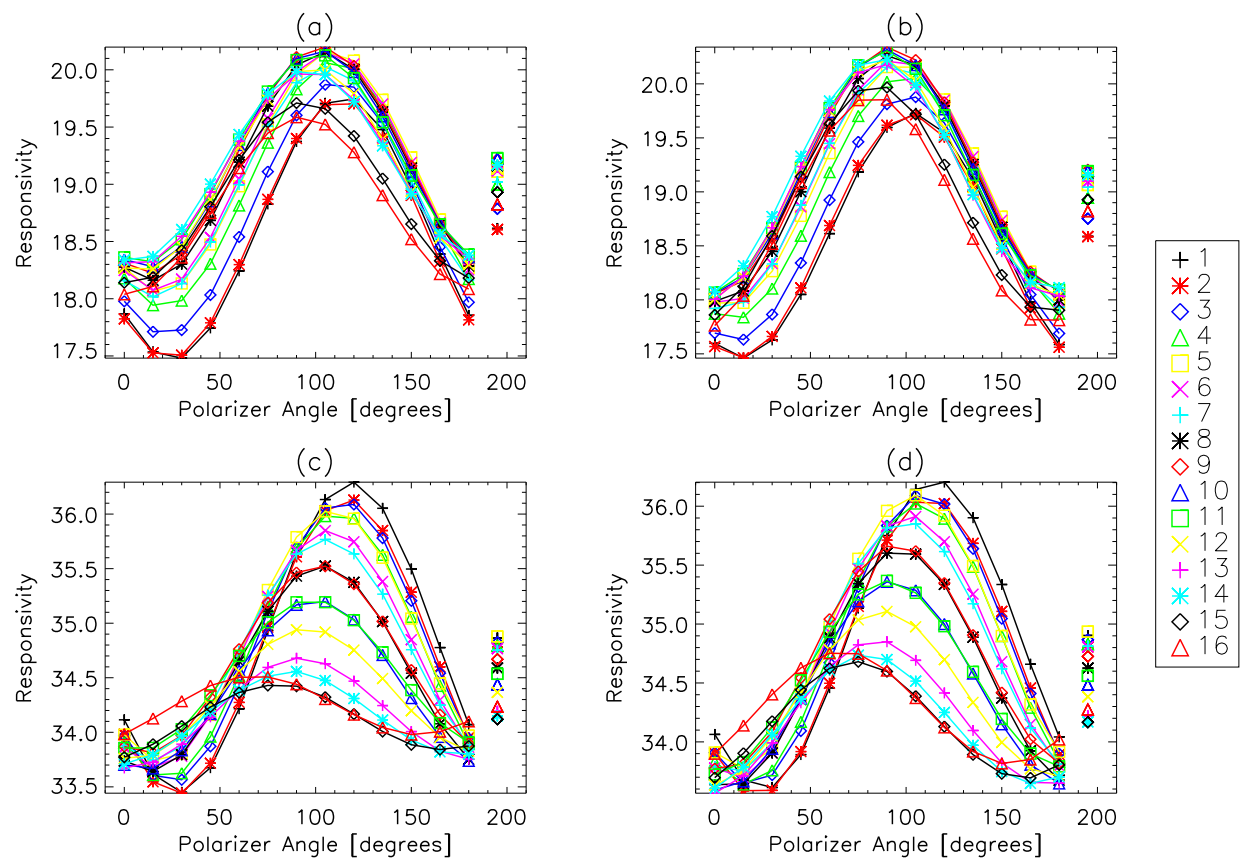

Figure 3. Plots are shown of the responsivity versus polarization state for M1 at -8 degrees scan angle (a), for M1 at +45 degrees scan angle (b), for M4 at - 8 degrees scan angle (c), and for M4 at +45 degrees scan angle (d). All data shown was collected using HAM side 1 and the responsivity is in units of $\left[d n /\left(\mathrm{W} / \mathrm{m}^{2} / \mathrm{sr} / \mu \mathrm{m}\right)\right]$. The legend defines the different symbol / color combinations which correspond to each detector. Note that the unconnected data at 195 degrees in each plot represents the responsivity derived from unpolarized data.

The calculated band M1 centroids and bandwidths derived from the flat spectrum ASR functions are shown in Figures 2(a) and (c); similarly, the M4 centroids and bandwidths are shown in Figures 2(b) and (d). Although Figure 2 only shows results from measurements made at -8 degrees scan angle, HAM side 1 , the results shown are indicative of all the test configurations measured. Note that the centroids and bandwidths derived from unpolarized data are plotted as the disconnected points at 195 degrees. The variation in centroid is small, up to $\sim 0.2 \mathrm{~nm}$ for M1 and $\sim 0.3 \mathrm{~nm}$ for M4. The bandwidth, however, varies by up to $\sim 1.5 \mathrm{~nm}$ for M1 and $\sim 1.7 \mathrm{~nm}$ for M4. There is also significant detector-to-detector dependence in M4 for the bandwidth, with the smallest variation being as low as $\sim 0.5 \mathrm{~nm}$. As noted above, the broadening or narrowing of the bandpass with polarization state leads to greater polarization sensitivity on the band edges and, as a result, larger band average polarization sensitivity. The average centroids determined from spectral testing ${ }^{7}$ were $411.1 \mathrm{~nm}$ and $556.4 \mathrm{~nm}$ for M1 and M4, respectively; the M1 values are consistent with spectral testing, but the M4 centroids are slightly higher in this work. The band average bandwidths derived from spectral testing were $17.5 \mathrm{~nm}$ (M1) and 18.4 $\mathrm{nm}$ (M4); in this case, the M1 values are slightly lower and the M4 bandwidths are consistent. Note that this may be the result of the spectral bandpass in this testing being under-sampled.

The responsivity for M1 derived using - 8 degrees scan angle, HAM side 1 data is shown in Figure 3(a) as a function of polarization angle. Similar responsivity graphs for M1 derived using +45 degrees scan angle, HAM side 1 data [Figure 3(b)], M4 derived using -8 degrees scan angle, HAM side 1 data [Figure 3(c)], and M4 derived using +45 degrees scan angle, HAM side 1 data [Figure 3(d)] are also shown. The responsivities for M4 derived using -8 degrees scan angle, HAM side 0 data are not shown, but are consistent with Figures 3(c) and (d). In addition, the responsivity derived from unpolarized data is shown as the unconnected data at 195 degrees in each sub-figure. The responsivities vary by up to $\sim 6.9 \%$ for $\mathrm{M} 1$ and $\sim 4.5 \%$ for M4, relative to the average value over polarization angles. This is consistent with the maximum band average polarization sensitivity. ${ }^{2}$ Again, there is some detector dependence for M1 (the responsivities vary by between $\sim 4.5$ and $\sim 6.9 \%$ at -8 degrees scan angle and between $\sim 6.3$ and $\sim 6.9 \%$ at +45 degrees scan angle) and higher detector dependence for M4 
(a)

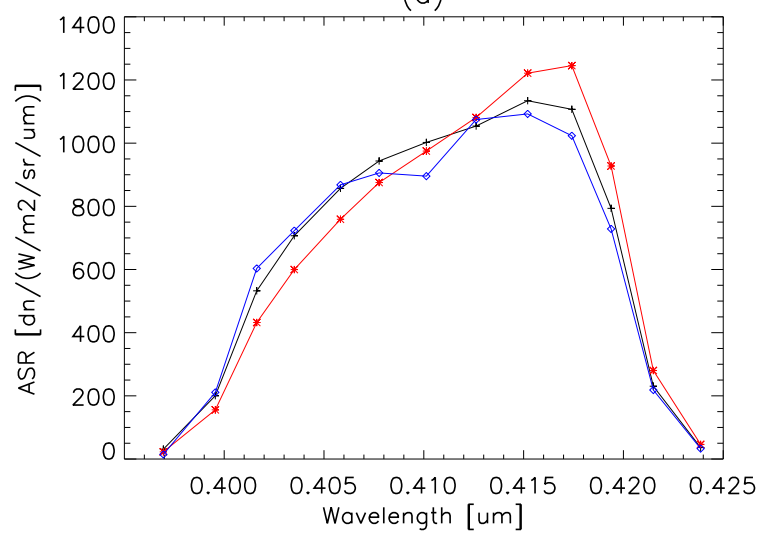

(b)

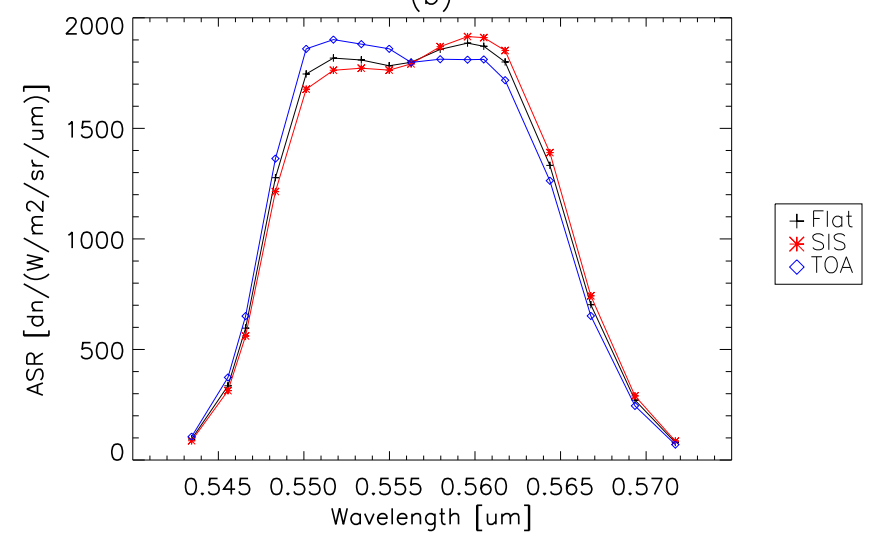

Figure 4. M1 ASR shown for detector 9 using data collected at -8 degrees scan angle, HAM side 1 (a) and M4 ASR shown for detector 9 using data collected at -8 degrees scan angle, HAM side 1 (b). The legend defines the different symbol / color combinations which correspond to the unpolarized $A S R$ for different input spectra.

(the responsivities vary by between $\sim 0.8$ and $\sim 4.5 \%$ at -8 degrees scan angle and between $\sim 1.4$ and $\sim 4.1 \%$ at +45 degrees scan angle). The observed detector dependence is also consistent with broadband polarization measurements. ${ }^{2}$ The band average responsivities derived from separate unpolarized radiometric measurements ${ }^{1}$ are 18.2 (M1) and 34.1 (M4), which are slightly lower than the unpolarized values reported here. This small discrepancy may result from the under-sampling for the spectral bandpass.

Examples of the unpolarized $A S R$ convolved with the SIS and TOA spectra are shown in Figure 4 for both M1 (a) and M4 (b) using data from detector 9, -8 degrees scan angle, HAM side 1. The SIS spectra is fairly smooth and increasing in these spectral regions from blue to red, while the TOA spectra decreases from blue to red and includes some structure. This causes the SIS spectra to shift the $A S R$ curve to longer wavelengths and the TOA spectra to shift the $A S R$ curve to shorter wavelengths. The structure in the TOA spectra is the source of the slight downturn in the ASR curve observed in the middle of the M1 bandpass.

The centroids derived from - 8 degrees scan angle, HAM side 1 data are graphed in Figure 5 for M1 convolved with the SIS (a) and TOA (b) spectra and for M4 convolved with the SIS (c) and TOA (d) spectra. The maximum variability with polarization state is about the same across the different input spectra and also when compared to the centroids derived from the flat spectra, shown in Figures 2(a) and (b). The band average M1 centroid determined from the unpolarized $A S R$ function increased by $\sim 0.65 \mathrm{~nm}$ from flat to SIS spectra and decreased from flat to TOA spectra by about $0.2 \mathrm{~nm}$. In the case of M4, the corresponding changes were $\sim 0.2$ and $\sim-0.3 \mathrm{~nm}$.

Figure 6 shows the bandwidths as a function of polarization state derived from the M1 $A S R$ curves convolved with the SIS (a) and TOA (b) spectra as well as the M4 ASR curves convolved with the SIS (c) and TOA (d) spectra (using data collected at -8 degrees scan angle, HAM side 1). The band average unpolarized bandwidths are $\sim 1.4 \mathrm{~nm}(\mathrm{M} 1)$ and $\sim 0.3 \mathrm{~nm}(\mathrm{M} 4)$ lower when the $A S R$ functions are convolved with the SIS spectrum and $\sim 0.2 \mathrm{~nm}$ higher (M1) and $\sim 0.1 \mathrm{~nm}$ lower (M4) when the $A S R$ functions are convolved with the TOA spectrum. In addition, the variability over polarization state differs from the flat input spectrum: the maximum bandwidth changes with polarization state are $\sim 0.9$ and $\sim 1.5 \mathrm{~nm}$ for M1 using the SIS and TOA spectra respectively and $\sim 2.1$ and $\sim 0.9 \mathrm{~nm}$ for M4 using the SIS and TOA spectra respectively. The bandwidth as calculated from Eq. (4) will vary between different input spectra solely due to changes in $\max [\operatorname{ASR}(\lambda, \alpha)]_{\lambda}$ [the responsivity is invariant under the convolution as defined in Eq. (5)]. Changes in the maximum $A S R$ value across polarization state leads to the bandwidth estimates deviating from a clean two cycle oscillation as seen in Figures 6(a) and (d). This is believed to result largely from the under-sampling of the bandpass during TSIRCUS testing. Furthermore, the bandwidths derived from unpolarized data should be roughly equivalent to the average over all the bandwidths derived from polarized data; however, as seen in Figures 6(a) and (d), this 
(a)

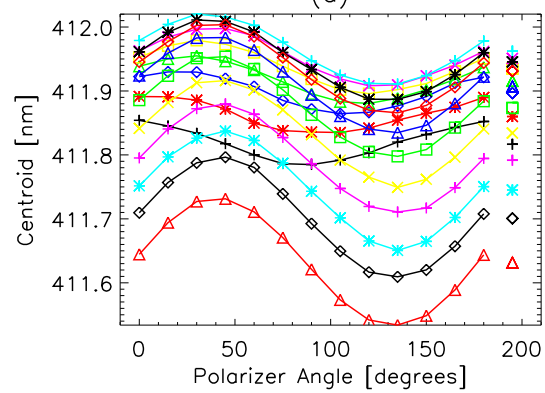

(c)

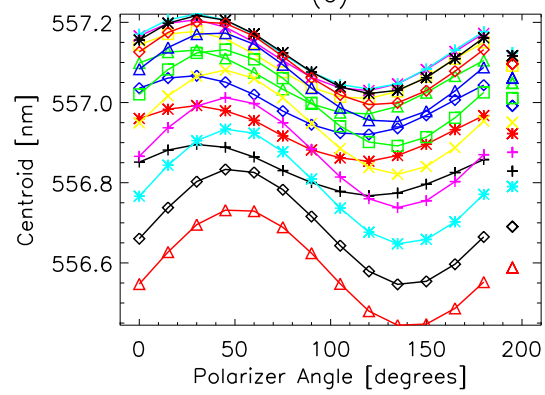

(b)

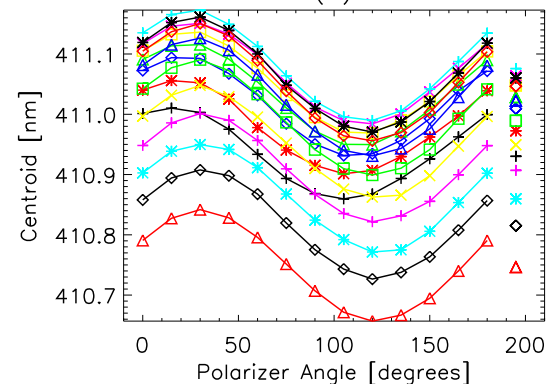

(d)

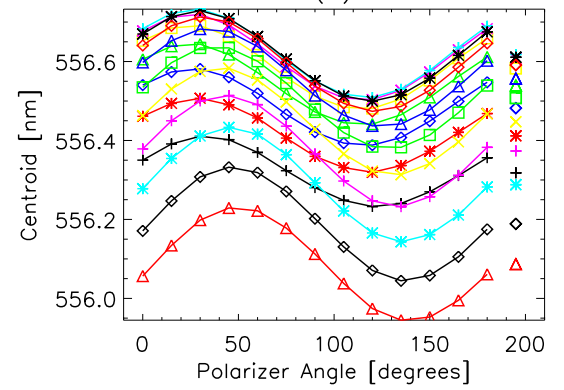

Figure 5. Plots of the band centroid versus polarization state for M1 using the SIS spectrum (a), M1 using the TOA spectrum (b), M4 using the SIS spectrum (c), M4 using the TOA spectrum (d). All data shown is from -8 degrees scan angle, HAM side 1. The legend defines the different symbol / color combinations which correspond to each detector. Note that the unconnected data at 195 degrees in each plot represents the centroid derived from unpolarized data.

is not always the case.

\section{CONCLUSIONS}

Monochromatic polarization measurements of JPSS J1 VIIRS using the NIST T-SIRCUS were made under a limited set of conditions and for a limited number of spectral bands. These measurements were used to construct spectral responsivity functions for all measured polarization states. From these spectral responsivity functions, the variation in centroid, bandwidth, and responsivity with polarization state was investigated. Results indicated that the variation in centroid was small (less than $\sim 0.3 \mathrm{~nm}$ for all cases) and that the variation in bandwidth was relatively large (up to about $1.7 \mathrm{~nm}$ ). The responsivity changed by up to $\sim 6.9 \%$ for $\mathrm{M} 1$ and $\sim 4.5 \%$ for $\mathrm{M} 4$, with some significant detector dependence observed for M4; this is consistent with the polarization sensitivity observed during broadband measurements. ${ }^{2}$ In addition, the spectral responsivity functions (derived using a flat spectrum) were convolved with two model input spectra: a SIS spectrum to simulate pre-launch testing and a TOA spectrum to estimate the possible on-orbit effects. For most cases, the SIS and TOA spectra changed the centroid and bandpass by less than $0.3 \mathrm{~nm}$; however, the SIS spectra did have a more sizable impact on M1 with the centroid varying by up to $0.65 \mathrm{~nm}$ and the bandwidth changing by as much as $1.4 \mathrm{~nm}$.

\section{ACKNOWLEDGMENTS}

The authors would like to acknowledge the following: Steve Brown and Keith Lykke from NIST and Brendan McAndrew from NASA for setting up and operating the T-SIRCUS lasers during testing, Joel McCorkel from NASA for organizing the testing, and the Raytheon test team including Tung Wang for conducting the test. The above mentioned proved valuable information and support to the analysis presented in this work. 

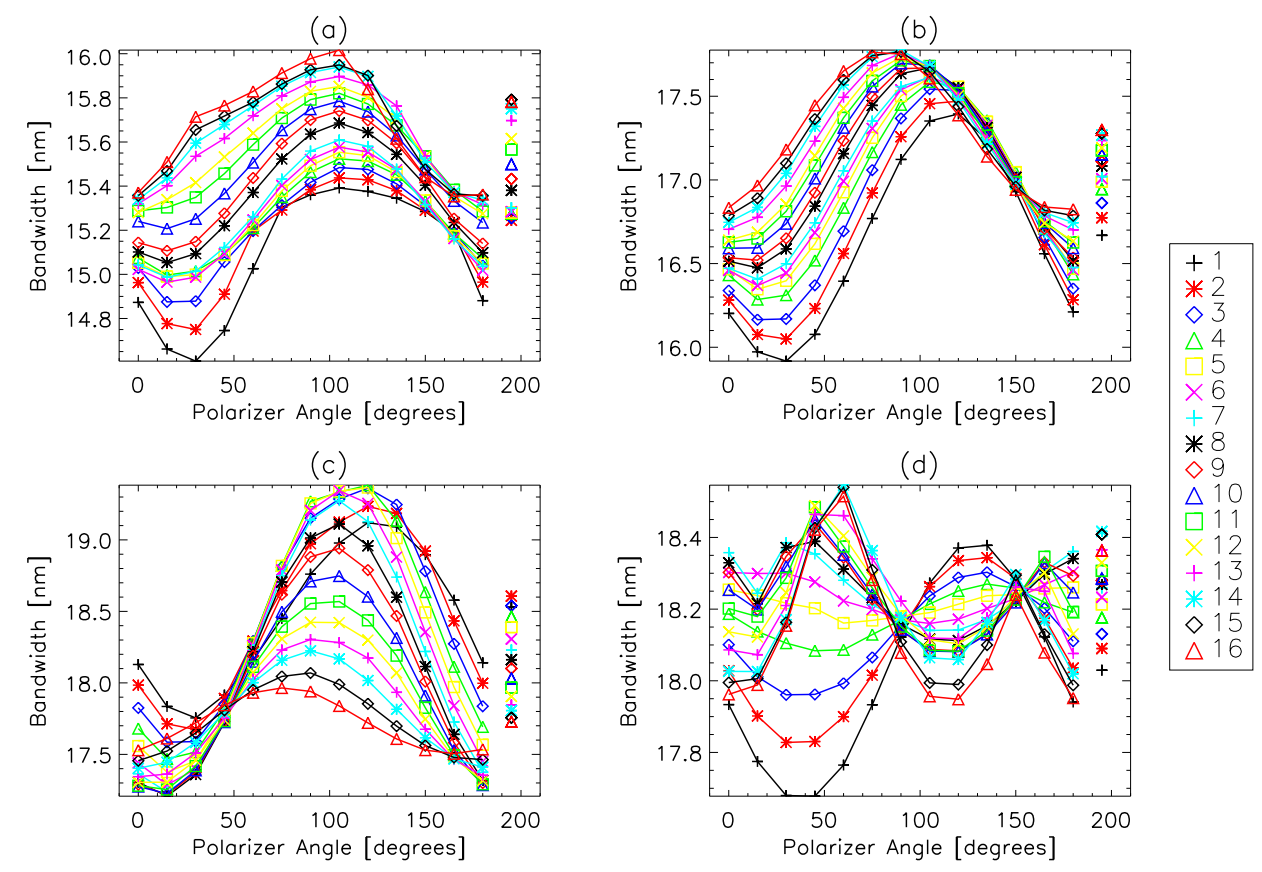

Figure 6. Plots of the bandwidth versus polarization state for M1 using the SIS spectrum (a), M1 using the TOA spectrum (b), M4 using the SIS spectrum (c), M4 using the TOA spectrum (d). All data shown is from -8 degrees scan angle, HAM side 1. The legend defines the different symbol / color combinations which correspond to each detector. Note that the unconnected data at 195 degrees in each plot represents the bandwidth derived from unpolarized data.

\section{REFERENCES}

[1] Oudrari, H., McIntire, J., Xiong, X., Butler, J., Efremova, B., Ji, Q., Lee, S., and Schwarting, T., "Preliminary assessment of JPSS-1 VIIRS pre-launch radiometric performance," Proc. of SPIE 9607 (2015). In press.

[2] Moyer, D., McIntire, J., Waluschka, E., Xiong, X., and DeLuccia, F., "JPSS-1 VIIRS pre-launch polarization testing and performance," IEEE T. Geosci. Remote (2015). In press.

[3] Waluschka, E., McCorkel, J., McIntire, J., Moyer, D., McAndrew, B., Brown, S. W., Lykke, K., Young, J. B., Fest, E., Butler, J., Wang, T. R., Monroy, E. O., Turpie, K., Meister, G., and Thome, K. J., "VIIRS polarization narrative," Proc. of SPIE 9607 (2015). In press.

[4] McIntire, J., Young, J. B., Moyer, D., Waluschka, E., Oudrari, H., and Xiong, X., "Analysis of JPSS J1 VIIRS polarization sensitivity using the NIST T-SIRCUS," Proc. of SPIE 9607 (2015). In press.

[5] Brown, S. W., Eppeldauer, G. P., and Lykke, K. R., "Facility for spectral irradiance and radiance responsivity calibrations using uniform sources," Appl. Opt. 45(32), 8218-8237 (2006).

[6] Barnes, R. A., Brown, S. W., Lykke, K. R., Guenther, B., Xiong, X., and Butler, J. J., "Comparison of two methodologies for calibrating satellite instruments in the visible and near infrared," Proc. of SPIE $\mathbf{7 8 6 2}$, $78620 \mathrm{C}(2010)$.

[7] Moeller, C., Schwarting, T., and Moyer, D., "JPSS J1 T-SIRCUS RSR," Unpublished (2015). 